\title{
RETRACTED ARTICLE: Advanced Business Model Innovation Supported by Artificial Intelligence, Deep Learning, Multi Business Model Patterns and a Multi Business Model Library
}

\author{
Peter Lindgren $^{1}$ (iD $\cdot$ Per Valter $^{1} \cdot$ Ramjee Prasad ${ }^{1}$
}

Published online: 26 March 2019

(c) Springer Science+Business Media, LLC, part of Springer Nature 2019

The authors have retracted this article [1] because it has been previously published [2]. All authors agree to this retraction.

[1] Lindgren, P., Valter, P. \& Prasad, R. Wireless Pers Commun (2019). https://doi. org/10.1007/s11277-019-06260-X

[2] Valter, P., Lindgren, P. \& Prasad, R. Wireless Pers Commun (2018) 100: 97. https:// doi.org/10.1007/s11277-018-5612-X

Electronic supplementary material The online version of this article (https://doi.org/10.1007/s1127 7-019-06260-x) contains the full text of the retracted article as electronic supplementary material.

Peter Lindgren

peterli@btech.au.dk

Per Valter

valter@btech.au.dk

Ramjee Prasad

ramjee@btech.au.dk

1 Business Development and Technology, Aarhus University, Aarhus, Denmark 